\title{
Association between oligo-residual disease and patterns of failure during EGFR-TKI treatment in EGFR-mutated non-small cell lung cancer: a retrospective study
}

Taichi Miyawaki ${ }^{1,2}$, Hirotsugu Kenmotsu ${ }^{1 *}$, Hiroaki Kodama ${ }^{1}$, Naoya Nishioka ${ }^{1}$, Eriko Miyawaki ${ }^{1}$, Nobuaki Mamesaya ${ }^{1}$, Haruki Kobayashi ${ }^{1}$, Shota Omori ${ }^{1}$, Ryo Ko ${ }^{1}$, Kazushige Wakuda ${ }^{1}$, Akira Ono ${ }^{1}$, Tateaki Naito ${ }^{1}$, Haruyasu Murakami ${ }^{1}$, Keita Mori ${ }^{3}$, Hideyuki Harada ${ }^{4}$, Masahiro Endo ${ }^{5}$, Kazuhisa Takahashi ${ }^{2}$ and

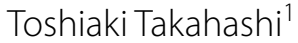

\begin{abstract}
Background: Local ablative therapy (LAT) may be beneficial for patients with epidermal growth factor receptor (EGFR) mutated non-small cell lung cancer (NSCLC) with oligo-residual disease after treatment with EGFR tyrosine kinase inhibitor (EGFR-TKI). However, this has not been fully established. This study aimed to evaluate the predominant progressive disease (PD) pattern limited to residual sites of disease after treatment with EGFR-TKI.

Methods: Patients with advanced EGFR-mutated NSCLC treated with EGFR-TKIs as first-line therapy were retrospectively analysed during a 7-year period. Oligo-residual disease was defined as the presence of $1-4$ lesions (including the primary site) at 3 months from the start of EGFR-TKI treatment. The predictive factors of PD patterns after EGFR-TKI treatment were evaluated.
\end{abstract}

Results: A total of 207 patients were included. Three months after the start of EGFR-TKI treatment, 66 patients (32\%) had oligo-residual disease. A total of 191 patients had PD, 60 with oligo-residual disease and 131 with non-oligoresidual disease. Regarding the pattern, 44 patients (73\%) with oligo-residual disease and 37 patients (28\%) with nonoligo-residual disease had PD limited to the residual sites. Multivariate logistic regression analysis at 3 months from the start of EGFR-TKI treatment revealed that oligo-residual disease $(P<0.001)$, the lack of residual central nervous system metastases $(P=0.032)$, and initial treatment with osimertinib $(P=0.028)$ were independent predictors of PD limited to residual disease sites.

Conclusions: This study provided a rationale for LAT to all sites of residual disease in patients with oligo-residual disease during EGFR-TKI treatment.

Keywords: Non-small-cell lung cancer, Oligo-residual disease, Failure pattern, EGFR-TKI, Osimertinib

*Correspondence: h.kenmotsu@scchr.jp

${ }^{1}$ Division of Thoracic Oncology, Shizuoka Cancer Center, 1007

Shimonagakubo, Nagaizumi-cho, Sunto-gun, Shizuoka 411-8777, Japan

Full list of author information is available at the end of the article

\begin{abstract}
Background
Epidermal growth factor receptor-tyrosine kinase inhibitors (EGFR-TKIs) have demonstrated clinical activity in the treatment of patients with EGFR-mutated non-small-cell lung cancer (NSCLC) [1], Several clinical trials have indicated the superiority of EGFR-TKIs
\end{abstract}


over conventional chemotherapy in terms of treatment efficacy, progression-free survival (PFS), and objective response rate in patients with EGFR-mutated NSCLC [1-3]. Furthermore, osimertinib, a third-generation EGFR-TKI, was associated with prolonged PFS and overall survival (OS) compared to first-generation EGFRTKIs, including erlotinib or gefitinib, in a phase III trial $[4,5]$. However, the development of resistance to EGFRTKIs remains an obstacle to achieving disease control and long-term survival [6].

Adding local ablative therapy (LAT) to standard systemic therapy could be a treatment option that provides high local control of residual disease in patients with oligometastatic NSCLC [7-9]. The European Society for Medical Oncology defines oligometastatic NSCLC as the presence of $1-3$ metastases and recommends the addition of LAT in these patients [10]. Previous studies have demonstrated that LAT of all sites of disease provided potential benefits in patients with synchronous oligometastatic NSCLC [11-13].

Progressive disease (PD) after first-line chemotherapy has been shown to be predominantly limited to the original site of disease, providing a rationale for the addition of LAT in patients with oligometastatic NSCLC $[14,15]$. In patients with EGFR-mutated NSCLC, almost half had PD limited to the original sites of disease after first-line treatment with EGFR-TKIs [16-18]. No significant association was found between synchronous oligometastatic disease and patterns of initial PD after EGFR-TKI treatment [18]. However, several studies have shown that LAT to all sites of residual disease demonstrated favourable PFS and OS in patients with oligo-residual disease [19-21].

The clinical features of oligo-residual disease are unknown. Furthermore, the patterns of initial PD after first-line treatment with EGFR-TKIs remain unclear in patients with oligo-residual disease. Our study aimed to evaluate the clinical impact of oligo-residual disease on patterns of PD after EGFR-TKI treatment and establish a rationale for adding LAT to all sites of oligo-residual disease.

\section{Methods}

\section{Patients}

The medical records of patients with stage IV EGFRmutated NSCLC who received EGFR-TKI first-line monotherapy at the Shizuoka Cancer Centre between January 2013 and December 2019 were retrospectively reviewed. This study's protocol was approved by our institutional ethics review board (approval number: J2020-177-20201) and was conducted in accordance with the Declaration of Helsinki. The need for informed consent was waived due to the retrospective nature of the study.
The collected data included age, sex, Eastern Cooperative Oncology Group performance status (ECOGPS), smoking history, EGFR mutation status, metastatic details (sites, number of organs, number of metastases), and type of EGFR-TKIs. EGFR gene mutations were evaluated using polymerase chain reaction amplification using commercially available assays. Age, ECOGPS, metastatic details, and the number of residual lesions were collected at 3 months \pm 6 weeks after the start of EGFR-TKI therapy. Two previous studies have shown that the median time from EGFR-TKI treatment initiation to maximum response was approximately 3 months $[16,22]$. Therefore, we evaluated the number of patients with residual disease at 3 months after EGFR-TKI treatment initiation, to evaluate the incidence of oligo-residual disease. Synchronous oligometastatic disease was defined as the presence of $1-3$ metastases $(1-4$ lesions including the primary site) at the time of NSCLC diagnosis $[12,14,23]$. Oligo-residual disease was defined as the presence of $1-4$ residual lesions, including the primary site, at 3 months after the start of EGFR-TKI (Fig. 1) $[19,23]$. Residual disease was defined as detectable lesions on imaging evaluation after 3 months of EGFRTKI therapy; moreover, lesions that had completely disappeared on computed tomography (CT) or magnetic resonance imaging (MRI) were not included in the definition of residual disease. Lesions that had previously been treated with local therapies, such as radiotherapy, were excluded from the definition of "residual disease" unless they had worsened. In all analysed patients, the residual lesions were independently evaluated by a thoracic oncologist and radiologist. Additionally, patients who received LAT before EGFR-TKI treatment were excluded from this study. Furthermore, no patient who developed oligo-residual disease after EGFR-TKI therapy induction underwent LAT.

Among the 308 eligible patients, those with EGFR exon20 insertion or initial T790M mutation $(n=9)$, those who did not continue EGFR-TKI therapy for 3 months ( $n=61,42$ due to disease progression, and 19 due to side effects), those without an efficacy evaluation at the previously mentioned time frame $(n=17)$, those transferred to other hospitals during the treatment period $(n=7)$ and other reasons $(n=7)$ were all excluded from this study (Fig. 2).

\section{Treatment and assessments}

The baseline disease stage was assessed using systemic and brain imaging. Metastatic lesions were independently evaluated by a thoracic oncologist and radiologist in all patients. Based on a randomised phase II study, any metastatic thoracic lymph nodes (N1 - N3), including those in the supraclavicular fossae, were collectively 


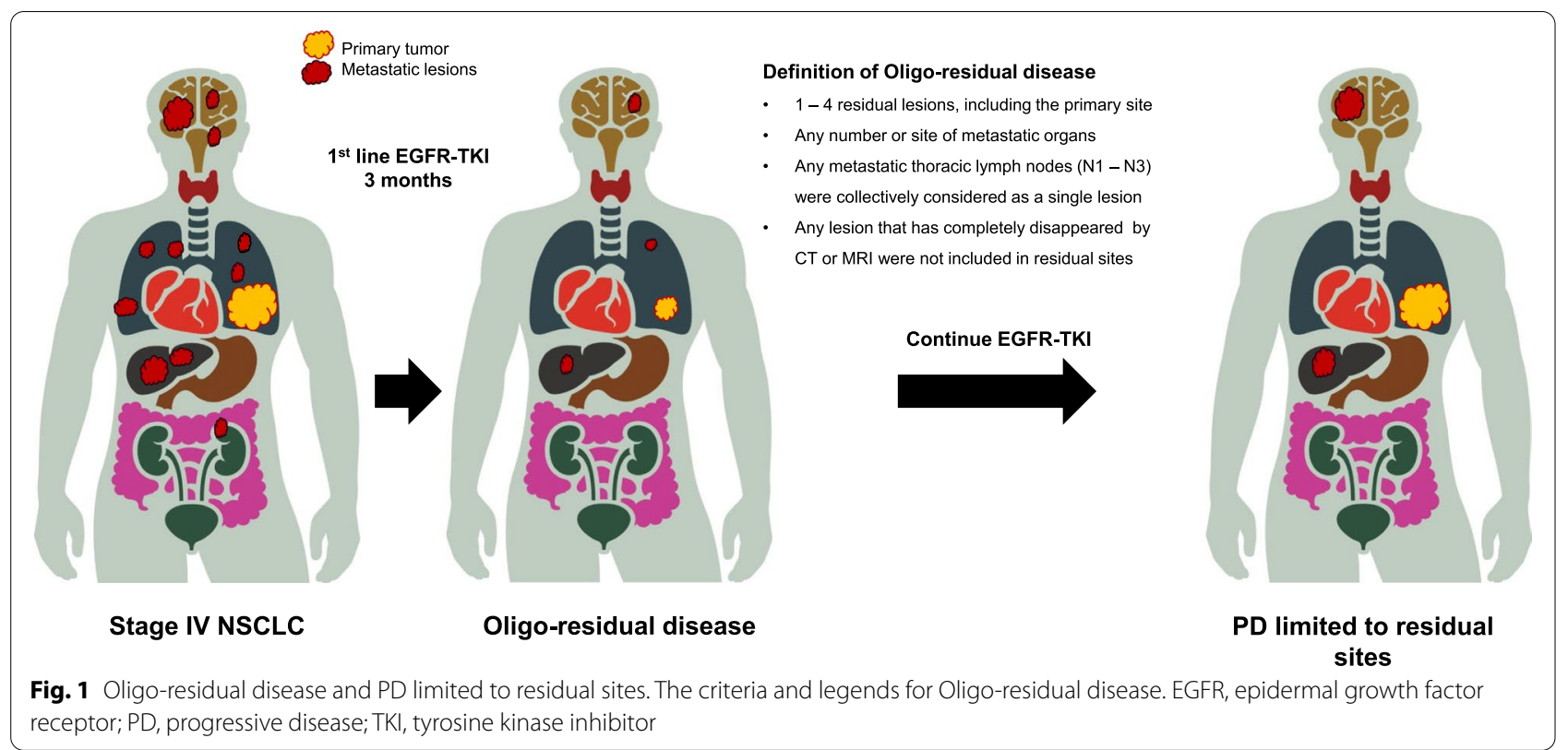

\section{Patient flow diagram}

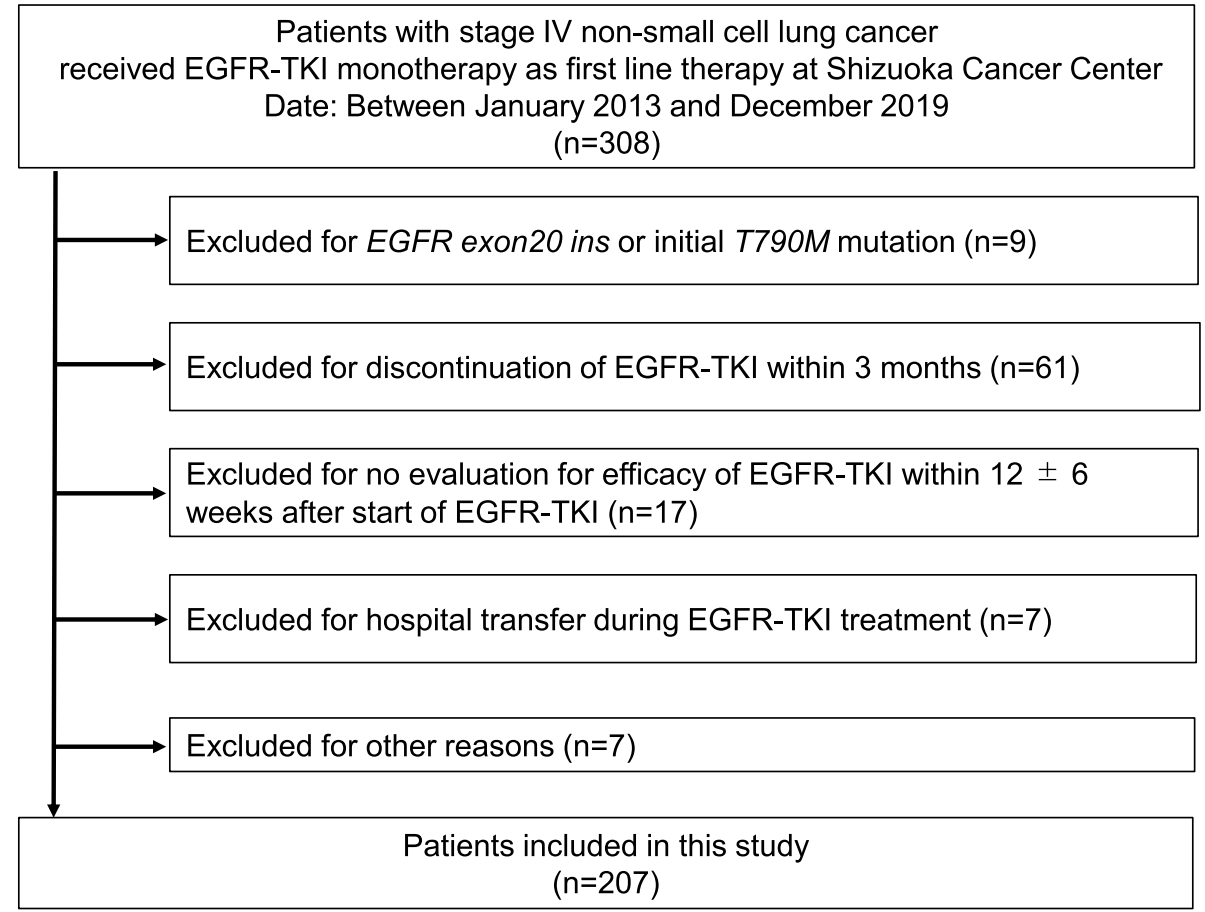

Fig. 2 Flowchart of included and excluded patients. EGFR, epidermal growth factor receptor; NSCLC, non-small cell lung cancer; TKI, tyrosine kinase inhibitor

considered as a single lesion [23]. PD was identified by reviewing follow-up radiological imaging, including CT and MRI, after the initiation of EGFR-TKI. Tumour responses were classified according to RECIST version 1.1 [24]. In most patients, chest and abdominal CT were performed every 6 to 12 weeks, and brain MRI was 
performed at the physician's discretion. PD patterns were classified as PD limited to residual sites of disease, PD at new sites, and PD at both sites (Fig. 1).

\section{Statistical analysis}

PFS was calculated from the residual disease evaluation to the first evidence of disease progression or death from any cause and estimated using the Kaplan-Meier method. The log-rank test was used to compare PFS between patients with oligo-residual disease and those with non-oligo residual disease. The end of the follow-up period was 31 December 2020. All categorical variables were analysed using the chi-square test. Potential predictive factors for oligo-residual disease were assessed using univariate and multivariate analyses with a logistic regression model adjusted for patient characteristics at baseline. Potential predictive factors for PD limited to the residual sites were assessed using univariate and multivariate analyses with a logistic regression model adjusted for patient characteristics at 3 months from the start of EGFR-TKI therapy. For the univariate analyses, the covariates included age ( $\geq 75$ vs. $<75$ years), sex, smoking status, ECOG-PS $(0-1$ vs. $\geq 2)$, histology (adenocarcinoma vs. non-adenocarcinoma), EGFR mutation (exon 19 deletions / L858R point mutations vs. others), residual central nervous system (CNS) metastases (yes vs. no), number of residual metastatic organs $(0-1$ vs. $\geq$ 2), [25, 26] EGFR-TKI type (osimertinib vs. other EGFRTKIs), and oligo-residual disease. Factors with univariate $P$-values of $<0.1$, were subjected to multivariate analyses. Differences were considered statistically significant at $P$-values of $<0.05$. All analyses were performed using STATA version 14.0 (Stata Corp., Texas, USA).

\section{Results}

\section{Baseline patient characteristics}

A total of 207 patients were included in this study. The median patient age was 68 years $(34-88$ years). The majority of the patients had adenocarcinoma (94.7\%), ECOG-PS 0 - 1 (82.6\%), and sensitising EGFR mutations (95.1\%). Only $6 \%$ of patients had synchronous oligometastatic disease, and $87 \%$ of patients had 10 or more metastases at the start of EGFR-TKI treatment (Table 1).

\section{Oligo-residual disease}

Three months after the start of EGFR-TKI treatment, $32 \%(n=66)$ had oligo-residual disease. All 13 patients $(100 \%)$ with synchronous oligometastatic disease at baseline had oligo-residual disease. Out of the 194 patients with non-synchronous oligometastatic disease at baseline, 53 patients $(27 \%)$ showed oligo-residual disease at 3 months (Table 1 ).
Baseline clinical factors were investigated to identify the predictive factors of oligo-residual disease. The univariate logistic regression analysis demonstrated that ECOG-PS $0-1$ (Odds ratio [OR] $=6.41,95 \%$ confidence interval (CI) [1.89 - 21.77], $P=0.003)$ and the presence of only one metastatic organ $(\mathrm{OR}=4.10,95 \% \mathrm{CI}[2.18$ 7.72], $P<0.001$ ) were predictive factors for oligo-residual disease. The multivariate logistic regression analysis demonstrated that ECOG-PS $0-1(\mathrm{OR}=4.98,95 \% \mathrm{CI}$ [1.41 - 17.6], $P=0.013)$, the presence of only one metastatic organ $(\mathrm{OR}=3.59,95 \% \mathrm{CI}[1.86-6.93], P<0.001)$ and treatment with osimertinib $(\mathrm{OR}=2.33,95 \% \mathrm{CI}[1.06$ $-5.13], P=0.034$ ) were independent predictive factors of oligo-residual disease (Table 2).

\section{Comparison between the oligo-residual and non-oligo-residual disease}

The clinical characteristics of patients with oligo-residual disease and those with non-oligo-residual disease are summarised in Table 1. The distribution of ECOG-PS differed significantly between these two groups $(P=0.021)$. The predominant metastatic organs also differed significantly, as pleura, pulmonary, bone, and brain metastases were more frequently observed in patients with nonoligo-residual disease than in those with oligo-residual disease.

The median follow-up time for PFS was 16 months. The median PFS was 11.3 months (95\% CI [8.7 - 13.7]) in patients with oligo-residual disease and 9.1 months $(95 \%$ CI $[7.0-10.2])$ in patients with non-oligo-residual disease. There was no significant difference in PFS between patients with oligo-residual disease and those with nonoligo-residual disease (hazard ratio $=0.81,95 \%$ CI $[0.60$ $-1.10], P=0.183$ ) (Fig. 3).

\section{Pattern of PD}

A total of 191 patients (92\%) had PD, 60 of whom (91\%) had oligo-residual disease, and 131 (93\%) with nonoligo-residual disease. PD limited to residual sites was observed in 44 patients (73\%) with oligo-residual disease and 37 patients (28\%) with non-oligo-residual disease. The proportion of PD in the residual sites alone was significantly higher in patients with oligo-residual disease than in those with non-oligo-residual disease $(P<0.001)$ (Fig. 3).

The clinical factors at 3 months from the start of EGFRTKI treatment were investigated to identify the predictive factors of PD limited to residual sites. The univariate logistic regression analysis demonstrated that ECOG PS $0-1(\mathrm{OR}=2.96,95 \%$ CI $[1.26-6.94], P=0.012)$, the lack of CNS metastases (OR $=4.15,95 \%$ CI $[1.72$ 10.00], $P=0.002)$, residual metastatic organ number of $0-1(\mathrm{OR}=2.53,95 \% \mathrm{CI}[1.39-4.61], P=0.002)$, 
Table 1 Patient Characteristics at baseline and 3 months from EGFR-TKI

\begin{tabular}{|c|c|c|c|c|}
\hline \multirow[t]{2}{*}{ Characteristics $N=207(\%)$} & \multirow{2}{*}{$\begin{array}{l}\text { Baseline } \\
\text { Overall } \\
\mathrm{N}=207\end{array}$} & \multicolumn{3}{|c|}{3 months from EGFR-TKI } \\
\hline & & $\begin{array}{l}\text { Oligo-residual } \\
n=66(\%)\end{array}$ & $\begin{array}{l}\text { Non-Oligo } \\
n=141(\%)\end{array}$ & $P$ \\
\hline Median age (range) & $68(34-88)$ & $67(41-87)$ & $68(34-88)$ & 0.478 \\
\hline \multicolumn{5}{|l|}{ Sex } \\
\hline Male & $77(37)$ & $22(33)$ & $55(39)$ & 0.431 \\
\hline Female & $130(63)$ & $44(67)$ & $86(61)$ & \\
\hline \multicolumn{5}{|l|}{ ECOG-PS } \\
\hline 0 & $43(21)$ & $28(42)$ & $34(24)$ & 0.021 \\
\hline 1 & $128(62)$ & $36(55)$ & $90(64)$ & \\
\hline 2 & $26(12)$ & $2(3)$ & $15(11)$ & \\
\hline 3 & $10(5)$ & 0 & $2(1)$ & \\
\hline \multicolumn{5}{|l|}{ Smoking status } \\
\hline Ever & $109(53)$ & $34(52)$ & $64(45)$ & 0.411 \\
\hline Never & $98(47)$ & $32(48)$ & $77(55)$ & \\
\hline \multicolumn{5}{|l|}{ Histology } \\
\hline Adenocarcinoma & $196(95)$ & $61(92)$ & $135(96)$ & 0.321 \\
\hline Non-Adenocarcinoma & $11(5)$ & $5(8)$ & $6(4)$ & \\
\hline \multicolumn{5}{|l|}{ Type of EGFR mutation } \\
\hline Del 19 & $124(60)$ & $33(50)$ & $91(65)$ & 0.391 \\
\hline L858R & $73(35)$ & $30(45)$ & $43(30)$ & \\
\hline Other & $10(5)$ & $3(5)$ & $7(5)$ & \\
\hline \multicolumn{5}{|l|}{ Site of metastatic organs } \\
\hline Pleura & $122(59)$ & 0 & $92(65)$ & $<0.001$ \\
\hline Pulmonary & $114(55)$ & $17(26)$ & $63(45)$ & 0.009 \\
\hline Bone & $95(46)$ & $12(18)$ & $62(44)$ & $<0.001$ \\
\hline Brain & $71(34)$ & $6(9)$ & $32(23)$ & 0.018 \\
\hline Liver & $36(17)$ & $7(11)$ & $30(21)$ & 0.062 \\
\hline Adrenal grand & $24(12)$ & $5(8)$ & $19(14)$ & 0.217 \\
\hline Extra-thoracic lymph node & $23(11)$ & $4(6)$ & $12(9)$ & 0.538 \\
\hline Others & $13(6)$ & $2(3)$ & $10(7)$ & 0.244 \\
\hline \multicolumn{5}{|l|}{ Number of lesions } \\
\hline 1 & 0 & $17(8)$ & 0 & \\
\hline 2 & 0 & $19(9)$ & 0 & \\
\hline 3 & $3(1)$ & $20(10)$ & 0 & \\
\hline 4 & $10(5)$ & $10(5)$ & 0 & \\
\hline 5 & $5(3)$ & 0 & $2(1)$ & \\
\hline 6 & $5(3)$ & 0 & 0 & \\
\hline 7 & $1(0)$ & 0 & $2(1)$ & \\
\hline 8 & $3(1)$ & 0 & $1(0)$ & \\
\hline 9 & 0 & 0 & 0 & \\
\hline$\geq 10$ & $180(87)$ & 0 & $136(66)$ & \\
\hline \multicolumn{5}{|l|}{ Number of metastatic organs } \\
\hline 0 & 0 & $15(22)$ & 0 & \\
\hline 1 & $63(31)$ & $39(59)$ & $60(43)$ & \\
\hline 2 & $62(30)$ & $11(17)$ & $33(23)$ & \\
\hline 3 & $46(22)$ & $1(2)$ & $20(14)$ & \\
\hline 4 & $24(12)$ & 0 & $20(14)$ & \\
\hline 5 & $5(2)$ & 0 & $3(2)$ & \\
\hline 6 & $7(3)$ & 0 & $5(4)$ & \\
\hline
\end{tabular}


Table 1 (continued)

\begin{tabular}{|c|c|c|c|c|}
\hline \multirow[t]{2}{*}{ Characteristics $N=207$ (\%) } & \multirow{2}{*}{$\begin{array}{l}\text { Baseline } \\
\text { Overall } \\
\mathrm{N}=207\end{array}$} & \multicolumn{3}{|c|}{3 months from EGFR-TKI } \\
\hline & & $\begin{array}{l}\text { Oligo-residual } \\
n=66(\%)\end{array}$ & $\begin{array}{l}\text { Non-Oligo } \\
n=141(\%)\end{array}$ & $P$ \\
\hline \multicolumn{5}{|l|}{ EGFR-TKI treatment } \\
\hline Gefitinib & $96(46)$ & $30(45)$ & $66(47)$ & 0.132 \\
\hline Erlotinib & $49(24)$ & $11(17)$ & $38(27)$ & \\
\hline Afatinib & $24(12)$ & $8(22)$ & $16(11)$ & \\
\hline Osimertinib & $38(18)$ & $17(26)$ & $21(15)$ & \\
\hline
\end{tabular}

ECOG Eastern Cooperative Oncology Group, PS performance status, EGFR epidermal growth factor receptor, TKI tyrosine kinase inhibitor, CNS central nervous system, DEL 19 exon 19 deletions, L858R L858R point mutations

Table 2 Predictive factors of Oligo-residual disease using a logistic regression model adjusted for baseline patient characteristics

\begin{tabular}{|c|c|c|c|c|c|c|}
\hline \multirow{2}{*}{$\begin{array}{l}\text { Covariates } \\
\mathrm{N}=\mathbf{2 0 7}\end{array}$} & \multicolumn{3}{|c|}{ Univariate analysis } & \multicolumn{3}{|c|}{ Multivariate analysis } \\
\hline & OR & $95 \% \mathrm{Cl}$ & $P$-value & OR & $95 \% \mathrm{Cl}$ & $P$-value \\
\hline Age ( $<75$ years vs $\geq 75$ years) & 1.74 & $0.87-3.45$ & 0.114 & & & \\
\hline Sex (male vs female) & 1.29 & $0.69-2.36$ & 0.432 & & & \\
\hline ECOG performance status score ( $0-1$ vs $\geq 2$ ) & 6.41 & $1.89-21.77$ & 0.003 & 4.98 & $1.41-17.6$ & 0.013 \\
\hline Smoking status (ever vs never) & 1.28 & $0.71-2.29$ & 0.411 & & & \\
\hline Histology (adeno vs non-adeno) & 0.54 & $0.15-1.84$ & 0.327 & & & \\
\hline EGFR mutation (del19/L858R vs Others) & 0.64 & $0.23-1.77$ & 0.394 & & & \\
\hline No baseline CNS metastases & 1.00 & $0.53-1.85$ & 0.989 & & & \\
\hline Baseline number of metastatic organ ( 1 vs $\geq 2$ ) & 4.10 & 2.18-7.72 & $<0.001$ & 3.59 & $1.86-6.93$ & $<0.001$ \\
\hline Treatment with Osimertinib & 1.98 & $0.96-4.07$ & 0.063 & 2.33 & $1.06-5.13$ & 0.034 \\
\hline
\end{tabular}

Significant $P$-values are shown in bold type. OR odds ratio, Cl confidence interval, ECOG Eastern Cooperative Oncology Group, adeno adenocarcinoma, EGFR epidermal growth factor receptor, DEL 19 exon 19 deletions, L858R L858R point mutations, CNS central nervous system

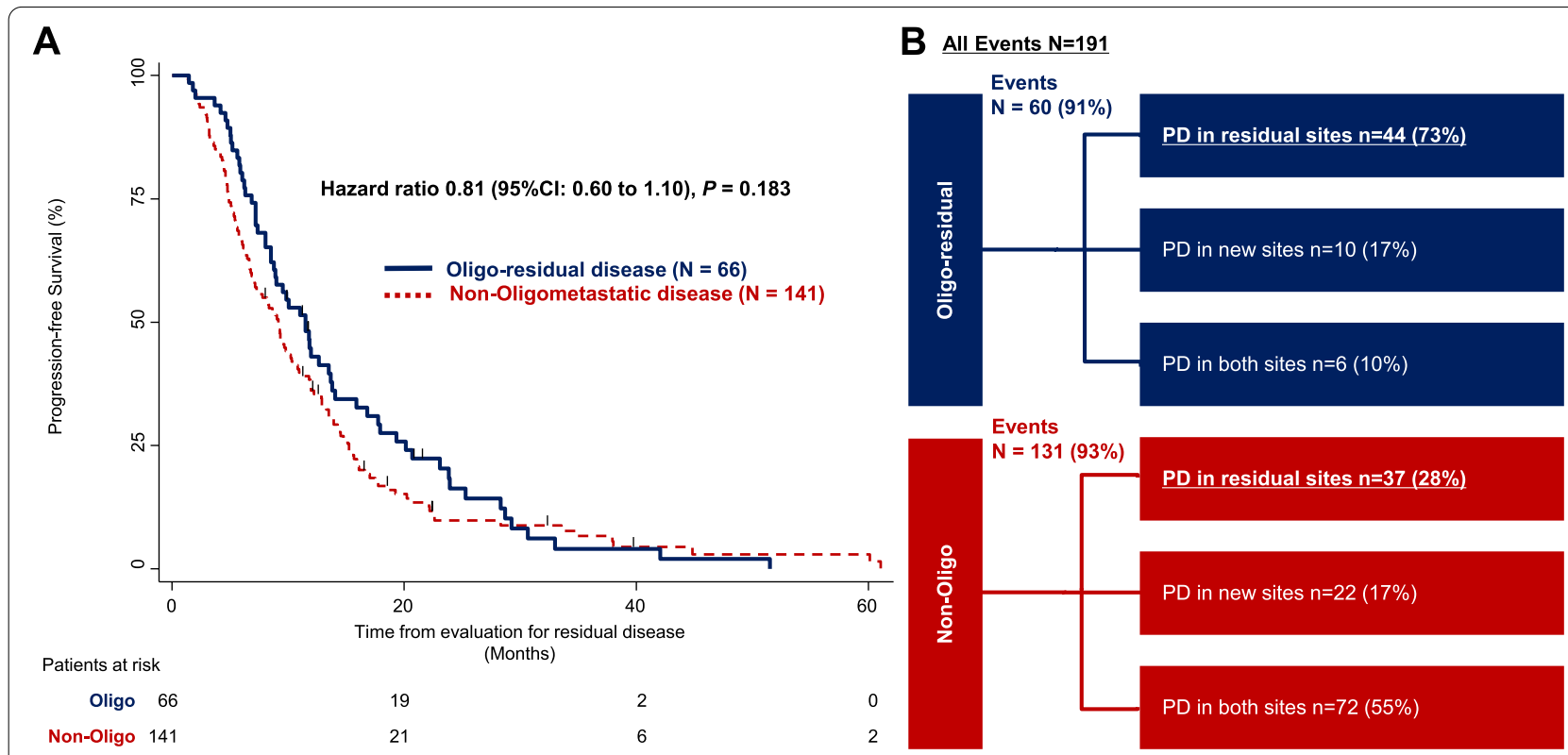

Fig. 3 A Kaplan-Meier curves for progression-free survival of oligo-residual and non-oligo-residual disease. The $P$-value was calculated using the log-rank test. The small vertical lines on the curve indicate patients who were censored. B Pattern of progressive disease. EGFR, epidermal growth factor receptor; PD, progressive disease; TKI, tyrosine kinase inhibitor 
oligo-residual disease $(\mathrm{OR}=6.98,95 \% \mathrm{CI}$ [3.51 - 13.24], $P<0.001$ ), and treatment with osimertinib were associated with PD limited to residual disease sites $(\mathrm{OR}=3.13$, 95\% CI [1.27 - 7.74], $P=0.013)$. The multivariate logistic regression analysis demonstrated that the lack of CNS metastases $(\mathrm{OR}=3.07,95 \% \mathrm{CI}[1.10-8.52], P=0.032)$, oligo-residual disease $(\mathrm{OR}=5.43,95 \% \mathrm{CI}$ [2.57 - 11.46], $P<0.001)$, and treatment with osimertinib $(\mathrm{OR}=3.24$, 95\% CI [1.13 - 9.25], $P=0.028)$ were independent predictors of PD limited to residual disease sites (Table 3).

Additionally, we investigated the clinical factors that were predictors of PD limited to the residual site prior to EGFR-TKI treatment initiation. Multivariate logistic regression analysis demonstrated that ECOG PS $0-1$ $(\mathrm{OR}=2.55,95 \% \mathrm{CI}[1.07-4.05], P=0.012)$, the absence of CNS metastases (OR $=1.95,95 \%$ CI [1.00-3.80], $P=0.049)$, and treatment with osimertinib were associated with $\mathrm{PD}$ limited to residual sites $(\mathrm{OR}=3.33,95 \% \mathrm{CI}$ [1.22 - 9.06], $P=0.018$ ). However, there was no significant association between the presence of oligometastatic disease or the number of organs involved in metastasis, and PD limited to residual sites (Supplementary Table 1).

\section{Discussion}

This is the first study that assesses the pattern of PD in patients with EGFR-mutated NSCLC using the residual disease evaluation at 3 months after the start of EGFRTKI treatment, including first-, second-, and third-generation EGFR-TKIs. Our study found that, after adjusting for other clinical factors, oligo-residual disease was an independent predictive factor of PD limited to the residual sites in patients with EGFR-mutated NSCLC. Analysing the pattern of PD after EGFR-TKI treatment might be essential to provide the rationale for LAT of all disease sites in patients with EGFR-mutated NSCLC, as well as in NSCLC patients without EGFR mutations [16, 18]. Our study also showed that more than $90 \%$ of patients had PD after EGFR-TKI. Acquired resistance remains a significant obstacle to achieving a durable response, even if the patients had oligo-residual disease response after EGFR-TKI treatment. Based on these data, adding consolidative LAT to all sites of residual disease at the time of response to EGFR-TKIs could potentially delay the time to progression or even improve survival outcomes in patients with oligo-residual disease.

The results of our study show that the majority of patients with oligo-residual disease have PD limited to residual sites after EGFR-TKI treatment. Preclinical studies have demonstrated that synchronous oligometastatic disease does not metastasise to other sites [27, 28]. EGFRTKI treatment might have caused EGFR-mutated polymetastatic disease to become oligometastatic, which was defined as oligo-residual disease. Furthermore, our study showed that only $6 \%$ of patients had synchronous oligometastatic disease before treatment, which increased to $32 \%$ after treatment. These findings suggest that EGFRTKI treatment for 3 months increased the number of patients eligible for LAT more than fivefold.

Limited to the small number of cases in our study, treatment with osimertinib was found to be an independent predictor of oligo-residual disease and PD limited to residual sites. No previous study has demonstrated the differences in PD patterns between osimertinib and other EGFR-TKIs. The FLAURA study showed that osimertinib was more effective than first-generation EGFR-TKIs, and suggested that the incidence of CNS relapses was lower in patients treated with osimertinib than in those treated with first-generation EGFR-TKIs (19\% vs. 43\%) [4, 5]. A recent retrospective study showed that osimertinib might delay the development of CNS metastasis compared to

Table 3 Predictive factors of PD limited to residual sites using a logistic regression model adjusted for patient characteristics

\begin{tabular}{|c|c|c|c|c|c|c|}
\hline \multirow{2}{*}{$\begin{array}{l}\text { Covariates } \\
N=191\end{array}$} & \multicolumn{3}{|c|}{ Univariate analysis } & \multicolumn{3}{|c|}{ Multivariate analysis } \\
\hline & OR & $95 \% \mathrm{Cl}$ & $P$-value & OR & $95 \% \mathrm{Cl}$ & $P$-value \\
\hline Age ( $<75$ vs $\geq 75$ years) & 1.14 & $0.60-2.17$ & 0.669 & & & \\
\hline Sex (male vs female) & 1.05 & $0.58-1.90$ & 0.853 & & & \\
\hline ECOG performance status score ( $0-1$ vs $\geq 2$ ) & 2.96 & $1.26-6.94$ & 0.012 & 1.77 & $0.67-4.66$ & 0.249 \\
\hline Smoking status (ever vs never) & 1.65 & $0.92-2.95$ & 0.088 & 1.41 & $0.72-2.74$ & 0.315 \\
\hline Histology (adeno vs non-adeno) & 1.11 & $0.30-4.07$ & 0.874 & & & \\
\hline EGFR mutation (del19/L858R vs Others) & 2.60 & $0.69-9.76$ & 0.157 & & & \\
\hline No residual CNS metastases & 4.15 & $1.72-10.00$ & 0.002 & 3.07 & $1.10-8.52$ & 0.032 \\
\hline Number of residual metastatic organ (0-1 vs $\geq 2$ ) & 2.53 & $1.39-4.61$ & 0.002 & 1.02 & $0.48-2.14$ & 0.951 \\
\hline Oligo-residual disease & 6.98 & $3.51-13.24$ & $<0.001$ & 5.43 & 2.57-11.46 & $<0.001$ \\
\hline Treatment with Osimertinib & 3.13 & $1.27-7.74$ & 0.013 & 3.24 & $1.13-9.25$ & 0.028 \\
\hline
\end{tabular}

Significant $P$-values are shown in bold type. OR odds ratio, $C l$ confidence interval, ECOG Eastern Cooperative Oncology Group, adeno adenocarcinoma, EGFR epidermal growth factor receptor, CNS central nervous system m, del 19 exon 19 deletions, L858R L858R point mutations 
first-generation EGFR-TKIs [29, 30]. Intriguingly, the results of our study suggest that more effective EGFR-TKI treatment for CNS metastases may result in oligo-residual disease and PD limited to the residual sites. Additionally, the lack of residual CNS metastases at 3 months after the start of EGFR-TKI treatment was found to be an independent predictive factor of PD limited to residual sites in our study.

Previous studies have suggested that patients with brain metastases have a higher risk of developing newer brain metastases and have a poorer prognosis [31]. ECOG PS $0-1$ and having one metastatic organ at baseline were independent predictive factors of oligo-residual disease in patients with EGFR-mutated NSCLC. Previous studies have already shown that both were prognostic factors in EGFR-mutated NSCLC patients receiving EGFR-TKIs $[26,32]$.

There were some limitations to our study. Our analysis was limited by its retrospective nature and the inability to account for unknown confounders. A relatively small sample size has the potential to affect its statistical power. This study was conducted in a cohort from a single institution and was not validated independently. Moreover, the present study could not provide data on the molecular basis for the association of osimertinib use with the occurrence of residual disease and PD limited to residual sites. Future research should focus on the molecular mechanisms underlying these associations. Patients with few baseline metastases (oligometastasis) could not be included in the multivariate analysis because they all developed oligo-residual disease after EGFR-TKI treatment induction. Although not all patients had undergone comprehensive imaging at the time of disease progression, the patients were categorised based on all available imaging findings. Furthermore, this study included only patients who were able to continue EGFR-TKI therapy for 3 months, which may have resulted in selection bias. Additionally, since first-line treatment with osimertinib was approved in Japan in August 2018, the median follow-up period for these patients was 15.8 months, shorter than the 37.8 months for patients treated with first- and second-generation EGFR-TKIs. Thus, bias due to differences in follow-up periods cannot be ruled out. A randomised phase II study of osimertinib with or without local consolidation therapy for stage IV NSCLC (Clini calTrials.gov identifier: NCT03410043) is currently ongoing. The results of a prospective randomised study could confirm our hypothesis. Moreover, from a strictly scientific perspective, counting the number of residual lesions might be a crude surrogate marker of oligo-residual disease. Evaluation of the number of alleles with EGFR mutations in circulating cell-free DNA during EGFR-TKI treatment has been applied in several studies. Recent studies have suggested that minimal residual disease can detect changes in circulating cell-free DNA [33, 34, 35]. Thus, this has the potential to become a new method of identifying true oligo-residual disease [36].

\section{Conclusions}

In conclusion, our study revealed that oligo-residual disease was significantly associated with PD limited to residual sites after EGFR-TKI treatment in patients with EGFR-mutated NSCLC. These results provide a rationale for LAT of all disease sites in patients with EGFRmutated NSCLC and oligo-residual disease. Future studies should focus on the development of treatment strategies, including LAT, in patients with oligo-residual disease during treatment with EGFR-TKI.

\section{Abbreviations \\ CNS: Central nervous system; Cl: Confidence interval; ECOG-PS: Eastern Cooperative Oncology Group performance status; EGFR-TKI: Epidermal growth factor receptor-tyrosine kinase inhibitor; LAT: Local ablative therapy; NSCLC: Non-small-cell lung cancer; OR: Odds ratio; OS: Overall survival; PFS: Progression-free survival; PD: Progressive disease.}

\section{Supplementary Information}

The online version contains supplementary material available at https://doi. org/10.1186/s12885-021-08983-2.

Additional file 1: Supplementary Table 1. Predictive factors of PD limited to residual sites using a logistic regression model adjusted for patient characteristics at baseline.

Acknowledgements

We would like to thank Editage (https://www.editage.jp) for editing this manuscript.

\section{Authors' contributions}

TM and HK wrote the manuscript and researched data. HK reviewed and edited the manuscript. KM is a professional biostatistician and responsible for statistical analysis. All authors reviewed, approved the final version of the manuscript.

\section{Funding}

This research did not receive any specific grants from funding agencies in the public, commercial, or not-for-profit sectors.

\section{Availability of data and materials}

The datasets used and/or analysed during the current study are available from the corresponding author on reasonable request.

\section{Declarations}

Ethics approval and consent to participate

The study was approved by the institutional ethics review board of Shizuoka Cancer Centre (approval number: J2020-177-2020-1) and was conducted in accordance with the Declaration of Helsinki. The need for consent to participate was waived due to the retrospective nature of the study. 


\section{Consent for publication}

Not applicable.

\section{Competing interests}

HK (Kenmotsu) reports C/A and RF from Chugai Pharmaceutical Co, Ltd. C/A from Ono Pharmaceutical Co, Ltd., C/A from Boehringer Ingelheim, C/A from Eli Lilly K.K, C/A from Kyowa Hakko Kirin Co., Ltd., C/A from Bristol-Myers Squibb, C/A from MSD, C/A and RF from Novartis Pharma K.K., C/A and RF from Daiichi-Sankyo Co., Ltd., C/A and RF from AstraZeneca K.K., C/A from Pfizer, C/A from Taiho Pharma, outside the submitted work. NM reports C/A from AstraZeneca KK, Pfizer Japan, Inc., C/A from Chugai Pharmaceutical Co., Ltd., C/A and RF from Boehringer Ingelheim, C/A from MSD K.K., personal fees from TAIHO PHARMACEUTICAL CO., LTD., C/A from ONO PHARMACEUTICAL CO., LTD., outside the submitted work. HK (Kobayashi) reports C/A from Eli Lilly K.K, C/A from Taiho Pharmaceutical, personal fees from AstraZeneca, outside the submitted work. SO reports C/A from Chugai Pharmaceutical Co., Ltd., Ono Pharmaceutical, AstraZeneca K.K., Boehringer Ingelheim, Taiho Pharmaceutical, and MSD, which are unrelated to the submitted work. RK reports C/A and RF from Boehringer Ingelheim, C/A and RF from AstraZeneca, C/A from Taiho Pharmaceutical, Chugai Pharmaceutical, Ono Pharmaceutical, Pfizer, and Lilly, outside the submitted work. KW reports C/A and RF from Chugai Pharmaceutical Co., Ltd., C/A from Taiho Pharmaceutical, C/A from Boehringer Ingelheim, C/A from Eli Lilly K.K., C/A from Ono Pharmaceutical, C/A from MSD, $\mathrm{C} / \mathrm{A}$ and RF from AstraZeneca, RF from Novartis, RF from AbbVie, outside the submitted work. AO reports C/A from Taiho Pharmaceutical, C/A from Ono Pharmaceutical, C/A from Chugai Pharmaceutical Co., Ltd., C/A from Novartis Pharma K.K., outside the submitted work. HM reports C/A from AstraZeneca K.K., Ono Pharmaceutical, Bristol-Myers Squibb Japan, Chugai Pharmaceutical Co., Ltd., Pfizer Inc., Novartis Pharma K.K., Boehringer Ingelheim, Taiho Pharmaceutical, Eli Lilly K.K., and MSD, which are unrelated to the submitted work. HH reports C/A and RF from Daiichi Sankyo Pharmaceutical Co. AstraZeneca K.K., Brain Labo Co., and Chugai Pharmaceutical Co., and RF from the Japan Agency for Medical Research and Development and the National Cancer Centre Research and Development Fund, which are unrelated to the submitted work. ME reports C/A from Ono Pharmaceutical, C/A from AstraZeneca, C/A from Takeda Pharmaceutical Co., Ltd., C/A from Daiichi-Sankyo Co., Ltd., outside the submitted work. KT reports C/A and RF AstraZeneca K.K., Pfizer Japan, Inc., Eli Lilly K.K., MSD, and Boehringer Ingelheim as well as grants from Takeda Pharmaceutical Co., Ltd., Chugai Pharmaceutical Co., Ltd., Taiho Pharmaceutical Co., Ltd., KYORIN Pharmaceutical Co., Ltd., Ono Pharmaceutical Co., Ltd., GlaxoSmithKline Consumer Healthcare Japan K.K., SHIONOGI \& CO., LTD., and Novartis Pharma K.K., which are unrelated to the submitted work. TT reports C/A and RF from AstraZeneca KK, Pfizer Japan, Inc., Eli Lilly Japan K.K., Chugai Pharmaceutical Co., Ltd., Ono Pharmaceutical Co., Ltd., MSD K.K., Boehringer Ingelheim Japan, Inc., and Pfizer Japan, Inc., and Roche Diagnostics K.K., outside the submitted work. TM, HK (Kodama), NN, EM, TN, and, KM declare that they have no competing interests.

\section{Author details}

'Division of Thoracic Oncology, Shizuoka Cancer Center, 1007 Shimonagakubo, Nagaizumi-cho, Sunto-gun, Shizuoka 411-8777, Japan. ${ }^{2}$ Department of Respiratory Medicine, Juntendo University Graduate School of Medicine, Tokyo, Japan. ${ }^{3}$ Clinical Research Center, Shizuoka Cancer Center, Shizuoka, Japan. ${ }^{4}$ Radiation and Proton Therapy Center, Shizuoka Cancer Center, Shizuoka, Japan. ${ }^{5}$ Division of Diagnostic Radiology, Shizuoka Cancer Center, Shizuoka, Japan.

\section{Received: 16 July 2021 Accepted: 9 November 2021}

Published online: 19 November 2021

\section{References}

1. Mitsudomi T, Morita S, Yatabe Y, Negoro S, Okamoto I, Tsurutani J, et al. Gefitinib versus cisplatin plus docetaxel in patients with non-small-cell lung cancer harbouring mutations of the epidermal growth factor receptor (WJTOG3405): an open label, randomised phase 3 trial. Lancet Oncol. 2010:11:121-8. https://doi.org/10.1016/S1470-2045(09)70364-X.

2. Rosell R, Carcereny E, Gervais R, Vergnenegre A, Massuti B, Felip E, et al. Erlotinib versus standard chemotherapy as first-line treatment for European patients with advanced EGFR mutation-positive non-small-cell lung cancer (EURTAC): a multicentre, open-label, randomised phase 3 trial. Lancet Oncol. 2012;13:239-46. https://doi.org/10.1016/S1470-2045(11) 70393-X.

3. Maemondo M, Inoue A, Kobayashi K, Oizumi S, Isobe H, Gemma A, et al. Gefitinib or chemotherapy for non-small-cell lung cancer with mutated EGFR. N Engl J Med. 2010;362:2380-8. https://doi.org/10.1056/NEJMo a0909530.

4. Soria JC, Ohe Y, Vansteenkiste J, Reungwetwattana T, Chewaskulyong B, Lee $\mathrm{KH}$, et al. Osimertinib in untreated EGFR-mutated advanced nonsmall-cell lung cancer. N Engl J Med. 2018;378:113-25. https://doi.org/10. 1056/NEJMoa1713137.

5. Ramalingam SS, Vansteenkiste J, Planchard D, Cho BC, Gray JE, Ohe Y, et al Overall survival with osimertinib in untreated, EGFR-mutated advanced NSCLC. N Engl J Med. 2020;382:41-50. https://doi.org/10.1056/NEJMo a1913662.

6. Schmid S, Li JJN, Leighl NB. Mechanisms of osimertinib resistance and emerging treatment options. Lung Cancer. 2020;147:123-9. https://doi. org/10.1016/j.lungcan.2020.07.014.

7. Planchard D, Popat S, Kerr K. Metastatic non-small cell lung cancer: ESMO clinical practice guidelines for diagnosis, treatment and follow-up. Ann Oncol. 2018;29:iv192-237. https://doi.org/10.1093/annonc/mdy275.

8. Ettinger DS, Wood DE, Aggarwal C, Aisner DL, Akerley W, Bauman JR, et al. NCCN guidelines insights: non-small cell lung Cancer, version 1.2020. J Natl Compr Cancer Netw. 2019;17:1464-72. https://doi.org/10.6004/ incen.2019.0059.

9. Guckenberger M, Lievens Y, Bouma AB, Collette L, Dekker A, deSouza $N M$, et al. Characterisation and classification of oligometastatic disease: a European Society for Radiotherapy and Oncology and European Organisation for Research and Treatment of Cancer consensus recommendation. Lancet Oncol. 2020;21:e18-28. https://doi.org/10.1016/ S1470-2045(19)30718-1.

10. Novello S, Barlesi F, Califano R, Cufer T, Ekman S, Levra MG, et al. Metastatic non-small cell lung cancer: ESMO clinical practice guidelines for diagnosis, treatment and follow-up. Ann Oncol. 2016;27:v1-v27. https:// doi.org/10.1093/annonc/mdw326.

11. lyengar P, Wardak Z, Gerber DE, Tumati V, Ahn C, Hughes RS, et al. Consolidative radiotherapy for limited metastatic non-small-cell lung cancer: a phase 2 randomized clinical trial. JAMA Oncol. 2018;4:e173501. https:// doi.org/10.1001/jamaoncol.2017.3501.

12. Gomez DR, Tang C, Zhang J, Blumenschein GR Jr, Hernandez M, Lee JJ, et al. Local consolidative therapy vs. maintenance therapy or observation for patients with oligometastatic non-small-cell lung cancer: long-term results of a multi-institutional, phase II, randomized study. J Clin Oncol. 2019;37:1558-65. https://doi.org/10.1200/JCO.19.00201.

13. Palma DA, Olson R, Harrow S, Gaede S, Louie AV, Haasbeek C, et al. Stereotactic ablative radiotherapy for the comprehensive treatment of oligometastatic cancers: long-term results of the SABR-COMET phase II randomized trial. J Clin Oncol. 2020;38:2830-8. https://doi.org/10.1200/ JCO.20.00818.

14. Miyawaki T, Wakuda K, Kenmotsu H, Miyawaki E, Mamsaya N, Kobayashi $\mathrm{H}$, et al. Proposing synchronous oligometastatic non-small-cell lung cancer based on progression after first-line systemic therapy. Cancer Sci. 2021;112:359-68. https://doi.org/10.1111/cas.14707.

15. Kim C, Hoang CD, Kesarwala AH, Schrump DS, Guha U, Rajan A. Role of local ablative therapy in patients with oligometastatic and oligoprogressive non-small cell lung cancer. J Thorac Oncol. 2017;12:179-93. https:// doi.org/10.1016/j.jtho.2016.10.012.

16. Al-Halabi H, Sayegh K, Digamurthy SR, Niemierko A, Piotrowska Z, Willers $\mathrm{H}$, et al. Pattern of failure analysis in metastatic EGFR-mutant lung cancer treated with tyrosine kinase inhibitors to identify candidates for consolidation stereotactic body radiation therapy. J Thorac Oncol. 2015;10:16017. https://doi.org/10.1097/JTO.0000000000000648.

17. Li XY, Zhu XR, Zhang CC, Yu W, Zhang B, Shen TL, et al. Analysis of progression patterns and failure sites of patients with metastatic lung adenocarcinoma with EGFR mutations receiving first-line treatment of tyrosine kinase inhibitors. Clin Lung Cancer. 2020;21:534. https://doi.org/10.1016/j. cllc.2020.04.004.

18. Patel SH, Rimner A, Foster A, Zhang Z, Woo KM, Yu HA, et al. Patterns of initial and intracranial failure in metastatic EGFR-mutant non-small cell lung cancer treated with erlotinib. Lung Cancer. 2017;108:109-14. https:// doi.org/10.1016/j.lungcan.2017.03.010. 
19. Chan OSH, Lam KC, Li JYC, Choi FPT, Wong CYH, Chang ATY, et al. ATOM: a phase II study to assess efficacy of preemptive local ablative therapy to residual oligometastases of NSCLC after EGFR TKI. Lung Cancer. 2020;142:41-6. https://doi.org/10.1016/j.lungcan.2020.02.002.

20. Xu Q, Zhou F, Liu H, Jiang T, Li X, Xu Y, et al. Consolidative local ablative therapy improves the survival of patients with synchronous oligometastatic NSCLC harboring EGFR activating mutation treated with first-line EGFR-TKIs. J Thorac Oncol. 2018;13:1383-92. https://doi.org/10.1016/j. jtho.2018.05.019.

21. Elamin YY, Gomez DR, Antonoff MB, Robichaux JP, Tran H, Shorter MK, et al. Local consolidation therapy (LCT) after first line tyrosine kinase inhibitor (TKI) for patients with EGFR mutant metastatic non-small-cell lung cancer (NSCLC). Clin Lung Cancer. 2019;20:43-7. https://doi.org/10. 1016/j.cllc.2018.09.015.

22. Gomez DR, Blumenschein GR, Lee JJ, Hernandez M, Ye R, Camidge DR, et al. Local consolidative therapy versus maintenance therapy or observation for patients with oligometastatic non-small-cell lung cancer without progression after first-line systemic therapy: a multicentre, randomised, controlled, phase 2 study. Lancet Oncol. 2016;17:1672-82. https://doi. org/10.1016/S1470-2045(16)30532-0.

23. Guo T, Ni J, Yang X, Li Y, Li Y, Zou L, et al. Pattern of recurrence analysis in metastatic EGFR-mutant NSCLC treated with osimertinib: implications for consolidative stereotactic body radiation therapy. Int J Radiat Oncol Biol Phys. 2020;107:62-71. https://doi.org/10.1016/j.ijrobp.2019.12.042.

24. Eisenhauer EA, Therasse P, Bogaerts J, Schwartz LH, Sargent D, Ford R, et al. New response evaluation criteria in solid tumours: revised RECIST guideline (version 1.1). Eur J Cancer. 2009;45:228-47. https://doi.org/10. 1016/j.ejca.2008.10.026.

25. He YY, Zhang XC, Yang JJ, Niu FY, Zeng Z, Yan HH, et al. Prognostic significance of genotype and number of metastatic sites in advanced non-small-cell lung cancer. Clin Lung Cancer. 2014;15:441-7. https://doi. org/10.1016/j.cllc.2014.06.006.

26. Paralkar VR, LiT, Langer CJ. Population characteristics and prognostic factors in metastatic non-small-cell lung cancer: a fox Chase Cancer Center retrospective. Clin Lung Cancer. 2008;9:116-21. https://doi.org/10.3816/ CLC.2008.n.018.

27. Yachida S, Jones S, Bozic I, Antal T, Leary R, Fu B, et al. Distant metastasis occurs late during the genetic evolution of pancreatic cancer. Nature. 2010;467:1114-7. https://doi.org/10.1038/nature09515.

28. Goss PE, Chambers AF. Does tumour dormancy offer a therapeutic target? Nat Rev Cancer. 2010;10:871-7. https://doi.org/10.1038/nrc2933.
29. Zhou Y, Wang B, Qu J, Yu F, Zhao Y, Li S, et al. Survival outcomes and symptomatic central nervous system (CNS) metastasis in EGFR-mutant advanced non-small cell lung cancer without baseline CNS metastasis: Osimertinib vs. first-generation EGFR tyrosine kinase inhibitors. Lung Cancer. 2020;150:178-85. https://doi.org/10.1016/j.lungcan.2020.10.018.

30. Heon S, Yeap BY, Britt GJ, Costa DB, Rabin MS, Jackman DM, et al. Development of central nervous system metastases in patients with advanced non-small cell lung cancer and somatic EGFR mutations treated with gefitinib or erlotinib. Clin Cancer Res. 2010;16:5873-82. https://doi.org/10. 1158/1078-0432.CCR-10-1588.

31. Su PL, Wu YL, Chang WY, Ho CL, Tseng YL, Lai WW, et al. Preventing and treating brain metastases with three first-line EGFR-tyrosine kinase inhibitors in patients with EGFR mutation-positive advanced non-small cell lung cancer. Ther Adv Med Oncol. 2018;10:1758835918797589. https:// doi.org/10.1177/1758835918797589.

32. Inoue A, Kobayashi K, Usui K, Maemondo M, Okinaga S, Mikami I, et al. First-line gefitinib for patients with advanced non-small-cell lung cancer harboring epidermal growth factor receptor mutations without indication for chemotherapy. J Clin Oncol. 2009;27:1394-400. https://doi.org/ 10.1200/JCO.2008.18.7658.

33. Blumenthal GM, Bunn PA, Chaft JE, McCoach CE, Perez EA, Scagliotti GV, et al. Current status and future perspectives on neoadjuvant therapy in lung cancer. J Thorac Oncol. 2018;13:1818-31. https://doi.org/10.1016/j. jtho.2018.09.017.

34. Abbosh C, Birkbak NJ, Wilson GA, Jamal-Hanjani M, Constantin T, Salari R, et al. Phylogenetic ctDNA analysis depicts early-stage lung cancer evolution. Nature. 2017;545:446-51. https://doi.org/10.1038/nature22364.

35. I wama E, Sakai K, Azuma K, Harada T, Harada D, Nosaki K, et al. Monitoring of somatic mutations in circulating cell-free DNA by digital PCR and next-generation sequencing during afatinib treatment in patients with lung adenocarcinoma positive for EGFR activating mutations. Ann Oncol. 2017;28:136-41. https://doi.org/10.1093/annonc/mdw531.

36. Wu YL, Sequist LV, Hu CP, Feng J, Lu S, Huang Y, et al. EGFR mutation detection in circulating cell-free DNA of lung adenocarcinoma patients: analysis of LUX-lung 3 and 6. Br J Cancer. 2017;116:175-85. https://doi. org/10.1038/bjc.2016.420.

\section{Publisher's Note}

Springer Nature remains neutral with regard to jurisdictional claims in published maps and institutional affiliations.
Ready to submit your research? Choose BMC and benefit from:

- fast, convenient online submission

- thorough peer review by experienced researchers in your field

- rapid publication on acceptance

- support for research data, including large and complex data types

- gold Open Access which fosters wider collaboration and increased citations

- maximum visibility for your research: over $100 \mathrm{M}$ website views per year

At BMC, research is always in progress.

Learn more biomedcentral.com/submissions 